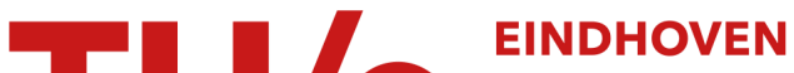 \\ UNIVERSITY OF \\ TECHNOLOGY
}

\section{Spin-polarized deuterium in magnetic traps}

Citation for published version (APA):

Koelman, J. M. V. A., Stoof, H. T. C., Verhaar, B. J., \& Walraven, J. T. M. (1987). Spin-polarized deuterium in magnetic traps. Physical Review Letters, 59(6), 676-679. https://doi.org/10.1103/PhysRevLett.59.676

DOI:

10.1103/PhysRevLett.59.676

Document status and date:

Published: 01/01/1987

\section{Document Version:}

Publisher's PDF, also known as Version of Record (includes final page, issue and volume numbers)

\section{Please check the document version of this publication:}

- A submitted manuscript is the version of the article upon submission and before peer-review. There can be important differences between the submitted version and the official published version of record. People interested in the research are advised to contact the author for the final version of the publication, or visit the $\mathrm{DOI}$ to the publisher's website.

- The final author version and the galley proof are versions of the publication after peer review.

- The final published version features the final layout of the paper including the volume, issue and page numbers.

Link to publication

\section{General rights}

Copyright and moral rights for the publications made accessible in the public portal are retained by the authors and/or other copyright owners and it is a condition of accessing publications that users recognise and abide by the legal requirements associated with these rights.

- Users may download and print one copy of any publication from the public portal for the purpose of private study or research.

- You may not further distribute the material or use it for any profit-making activity or commercial gain

- You may freely distribute the URL identifying the publication in the public portal.

If the publication is distributed under the terms of Article 25fa of the Dutch Copyright Act, indicated by the "Taverne" license above, please follow below link for the End User Agreement:

www.tue.nl/taverne

Take down policy

If you believe that this document breaches copyright please contact us at:

openaccess@tue.nl

providing details and we will investigate your claim. 


\title{
Spin-Polarized Deuterium in Magnetic Traps
}

\author{
J. M. V. A. Koelman, H. T. C. Stoof, and B. J. Verhaar \\ Department of Physics, Eindhoven University of Technology, NL-5600 MB Eindhoven, The Netherlands \\ and \\ J. T. M. Walraven \\ Natuurkundig Laboratorium, Universiteit van Amsterdam, NL-1018 XE Amsterdam, The Netherlands \\ (Received 5 May 1987)
}

\begin{abstract}
We have calculated the spin-exchange two-body rate constants associated with the population dynamics of the hyperfine levels of atomic deuterium as a function of magnetic field in the Boltzmann zerotemperature limit. Results indicate that a gas of low-field-seeking deuterium atoms trapped in a static magnetic field minimum decays rapidly into an ultrastable gas of doubly spin-polarized deuterium. We also discuss the temperature dependence of various effects.
\end{abstract}

PACS numbers: $67.65 .+z, 76.90 .+d$

The interesting physics of the gaseous spin-polarized quantum systems has been primarily studied for the Bose system spin-polarized hydrogen and the Fermi system spin-polarized ${ }^{3} \mathrm{He} .{ }^{1}$ Although the extreme quantum nature of these spin-polarized systems has been established in a variety of experiments, the observation of degenerate quantum behavior so far has been out of reach of the experimentalists. For spin-down polarized hydrogen $(\mathrm{H} \downarrow)$ it was established ${ }^{2}$ that the critical density for BoseEinstein condensation (BEC) can only be approached up to a factor 10 because of the presence of a third-order recombination process which is dominant on the surfaces of the helium-covered sample cells. Also for gaseous ${ }^{3} \mathrm{He}$ the degeneracy regime $\left(T \ll T_{\mathrm{F}}\right.$, where $T_{\mathrm{F}}$ is the Fermi temperature) is far out of reach of experiments as a result of the relatively strong interaction effects which lead to the formation of the liquid state. ${ }^{1}$

Spin-polarized deuterium (D $\downarrow$ ) has attracted relatively little attention of the experimentalists as this gas was found to be much less stable than hydrogen. ${ }^{3,4}$ Nevertheless, the theoretical interest in this system is considerable. To establish the nature of the ground state of $\mathrm{D} \downarrow$ is a subtle problem which stimulated the use of the advanced methods of Fermi-fluid theory. ${ }^{5,6}$ It is predicted that the doubly polarized state ( $D \downarrow \downarrow$ ) should be gaseous down to $T=0 \mathrm{~K}$. Also the Landau parameters have been calculated, ${ }^{6}$ and extensive theoretical effort was used in the calculation of the transport properties of gaseous $\mathrm{D} \downarrow$ as a function of temperature. ${ }^{7}$

Recently, surface-free confinement schemes were proposed $^{8}$ which offer new prospects to observe BEC by studying spin-up polarized hydrogen $(\mathrm{H} \uparrow)$ in magnetic traps similar to those used for confining laser-cooled spin-polarized alkalis. ${ }^{9}$ In this Letter we show that $\mathrm{D} \uparrow \uparrow$ is especially suited for confinement in a minimum- $B$-field trap and may well prove to be the purest experimental realization of the nearly ideal degenerate Fermi gas in which to a large extent density and temper- ature can be controlled independently. As such it is a most interesting model system, allowing comparison with $a b$ initio theoretical results of any desired precision. This is in contrast to dense, strongly interacting Fermi systems such as nuclear matter, liquid ${ }^{3} \mathrm{He}$, and electron gases in metals.

We discuss the stability of $\mathrm{D} \uparrow$, a mixture of the hyperfine states $\delta, \epsilon$, and $\zeta$ (Fig. 1), confined in a static minimum- $B$-field trap. We calculate both spinexchange and dipolar two-body rate constants in the

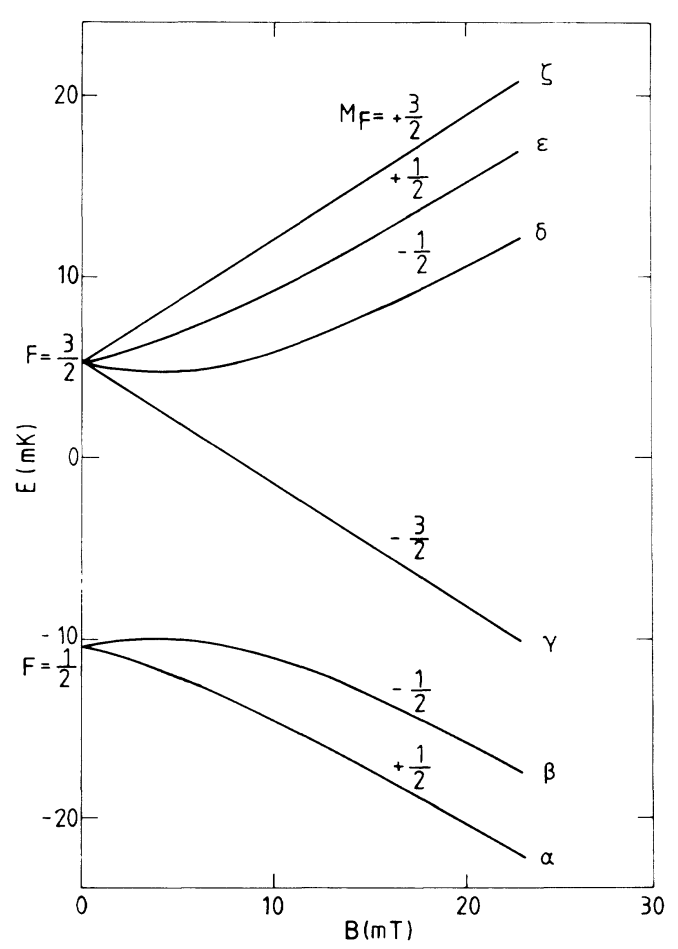

FIG. 1. Energies of the deuterium hyperfine states as a function of magnetic field. 
low-field, $T=0 \mathrm{~K} \mathrm{limit}$, and estimate the temperature and field dependence of these effects. We show that spin exchange causes $D \uparrow$ to decay rapidly towards the doubly polarized gas $D \uparrow \uparrow$ of only $\zeta$-state atoms. This gas may be cooled with a similar evaporative scheme as proposed ${ }^{8}$ for $\mathrm{H} \uparrow \uparrow$. However, in contrast to the hydrogen case where dipolar relaxation is predicted to be very fast and, to lowest order, independent of temperature, ${ }^{10}$ the stability of the fermion $\mathrm{D} \uparrow \uparrow$ against dipolar relaxation is expected to grow with decreasing temperature because of the absence of $s$-wave scattering, ultimately leading to an ultrastable stable. $\mathrm{D} \uparrow \uparrow$ is not only likely to be the most stable $B$-field-trappable spin-polarized system (including the alkalis), but may also be cooled well into the degeneracy regime. We briefly discuss how Fermi statistics affect the properties of $\mathrm{D} \uparrow \uparrow$. As a last point we address the stability of $\mathrm{D} \uparrow \uparrow$ against resonance recombination.

We first discuss the various relaxation processes in a gaseous mixture of $\delta$-, $\epsilon$-, and $\zeta$-state atoms (D $\uparrow$ ). The lifetimes of these low-magnetic-field seekers are primarily limited by inelastic spin-exchange relaxation events. Dipolar relaxation only becomes competitive at high magnetic fields ${ }^{10}(B \gtrsim 0.2 \mathrm{~T}$ for $\mathrm{D} \uparrow)$ and as such is not relevant in the current context. As in the hydrogen case an important exception to this rule is collisions between fully polarized atoms $(\zeta-\zeta$ collisions) which are unaffected by spin exchange. As a result of the low values of the relevant temperatures and Fermi-Dirac statistics, only low-energy $s$-wave scattering between atoms in antisymmetrical spin states occurs. We therefore calculated the eighteen rate constants corresponding to the allowed downward spin-exchange transitions between the fifteen antisymmetrized spin states $(\alpha \beta$ $-\beta \alpha) / \sqrt{2}, \ldots,(\epsilon \zeta-\zeta \epsilon) / \sqrt{2}$ for $s$-wave scattering in the Boltzmann zero-temperature limit. These rate constants can be expressed in terms of the two-body spinexchange $T$-matrix elements for vanishing kinetic energy in the incoming channel. When the splittings of the internal energy levels are not too large, we may also assume that the kinetic energy in the final channel vanishes. This approximation ${ }^{11}$ applies when the time interval in which the interaction associated with the internal degrees of freedom is interrupted by the exchange interaction is small compared with the time scales at which precessions associated with the internal-energy-level splittings take place. For low collision energies, this interruption time is determined by the time interval in which the colliding atoms can be localized within the interaction range: $\Delta t=\mu r_{0}^{2} / \hbar$ (with $\mu$ the reduced atomic mass and $r_{0}$ the range of the interaction). Using the above approximation, we find that the relaxation rates in the Boltzmann zero-temperature limit can be expressed in terms of the triplet and singlet scattering lengths $a_{T}$ and $a_{S}$ :

$$
G_{f i}=4 \pi v_{f}\left(\frac{a_{T}-a_{S}}{2}\right)^{2}\left|\left\langle f\left|P_{T}-P_{S}\right| i\right\rangle\right|^{2},
$$

in which $|i\rangle$ and $|f\rangle$ are normalized antisymmetric two-body spin states, $P_{T}\left(P_{S}\right)$ is the projection operator on the triplet (singlet) spin subspace, and $v_{f}=\left[2\left(E_{i}\right.\right.$ $\left.\left.-E_{f}\right) / \mu\right]^{1 / 2}$ is the relative velocity in the final spin channel.

Using the above expression in case of spin-exchange relaxation in atomic hydrogen with $a_{T}=1.34 a_{0}$ and $a_{S}=0.32 a_{0}$, we reproduce the values of the $\mathrm{H}+\mathrm{H}$ spinexchange relaxation rates obtained with a coupledchannels calculation ${ }^{10}$ within a few percent up to magnetic field strengths of $0.1 \mathrm{~T}$. In the case of atomic deuterium, we calculate $a_{T}=-6.8 a_{0}$ and $a_{S}=13.0 a_{0}$ and obtain the values for the eighteen zero-temperature spin-exchange relaxation rates displayed in Fig. 2. Interestingly enough for the present purposes, these rates are typically 2 orders of magnitude larger than the $\mathrm{H}+\mathrm{H}$ spin-exchange rates. ${ }^{10}$ This is due to the larger value for $\left|a_{T}-a_{S}\right|$ entering in the expression for the rates.

We now consider low-field-seeking deuterium atoms in a magnetic trap. If we use the notations $n_{\delta}, n_{\epsilon}$, and $n_{\zeta}$ for the densities of these states, and assume that all high-field-seeking atoms and a fraction $P$ of the lowfield-seeking atoms formed in inelastic spin-exchange events escape to a perfect adsorber outside the trapping region, the population dynamics of the various hyperfine

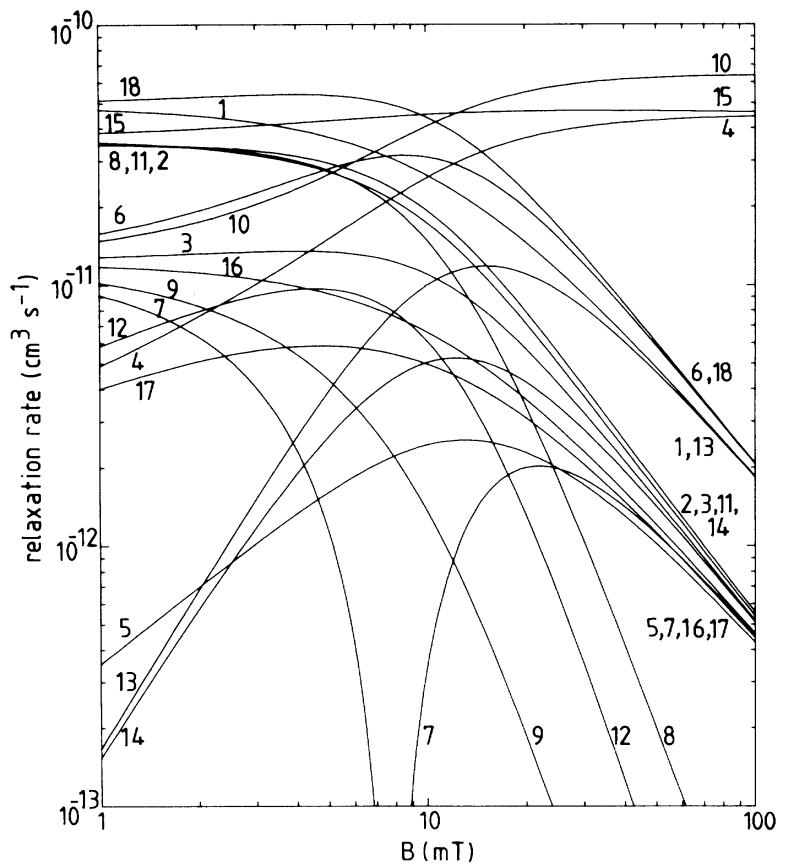

FIG. 2. The zero-temperature spin-exchange relaxation rates $G_{f i}$ as a function of magnetic field. The curves correspond to the following rates $i \rightarrow f: 1, \zeta \epsilon \rightarrow \zeta \alpha ; 2, \delta \zeta \rightarrow \alpha \epsilon ; 3$, $\delta \zeta \rightarrow \beta \zeta ; 4, \beta \epsilon \rightarrow \alpha \epsilon ; 5, \delta \epsilon \rightarrow \zeta \gamma ; 6, \delta \epsilon \rightarrow \beta \epsilon ; 7, \delta \epsilon \rightarrow \delta \alpha ; 8$, $\delta \epsilon \rightarrow \beta \alpha ; 9, \zeta \gamma \rightarrow \epsilon \beta ; 10, \zeta \gamma \rightarrow \delta \alpha ; 11, \zeta \gamma \rightarrow \beta \alpha ; 12, \beta \epsilon \rightarrow \alpha \delta$ $13, \beta \epsilon \rightarrow \beta \alpha ; 14, \alpha \delta \rightarrow \alpha \beta ; 15, \epsilon \gamma \rightarrow \delta \beta ; 16, \epsilon \gamma \rightarrow \alpha \gamma ; 17$, $\beta \delta \rightarrow \alpha \gamma, 18, \delta \zeta \rightarrow \beta \gamma$. 
levels is described by

$$
\begin{aligned}
& \dot{n}_{\delta}=-\left(G_{\alpha \epsilon, \delta \zeta}+G_{\beta \zeta, \delta \zeta}\right) n_{\delta} n_{\zeta}-\left(G_{\zeta \gamma, \delta \epsilon}+G_{\beta \epsilon, \delta \epsilon}+P G_{\delta \alpha, \delta \epsilon}+G_{\beta \alpha, \delta \epsilon}\right) n_{\delta} n_{\epsilon}, \\
& \dot{n}_{\epsilon}=-G_{\zeta \alpha, \zeta \epsilon} n_{\epsilon} n_{\zeta}-\left(G_{\zeta \gamma, \delta \epsilon}+P G_{\beta \epsilon, \delta \epsilon}+G_{\delta \alpha, \delta \epsilon}+G_{\beta \alpha, \delta \epsilon}\right) n_{\delta} n_{\epsilon}+(1-P) G_{\alpha \epsilon, \delta \zeta} n_{\delta} n_{\zeta}, \\
& \dot{n}_{\zeta}=-P G_{\zeta \alpha, \zeta \epsilon} n_{\epsilon} n_{\zeta}-\left(G_{\alpha \epsilon, \delta \zeta}+P G_{\beta \zeta, \delta \zeta}\right) n_{\delta} n_{\zeta}+(1-P) G_{\zeta \gamma, \delta \epsilon} n_{\delta} n_{\epsilon} .
\end{aligned}
$$

In general, a decay described by these equations yields a stable state consisting of one single hyperfine component. Which hyperfine state will survive depends on the relative magnitudes of the various decay rates, as well as on the escape probability $P$ and on the ratios between the initial populations. Substituting the above calculated relaxation rates, we find a preferential decay of $\delta$ and $\epsilon$ atoms. Hence, equal initial populations will lead to a trapped gas of $\zeta$ atoms ("doubly spin-polarized" deuteri$u m)$. The fraction of $\zeta$ atoms which survive the spinexchange decay process when starting with equal initial populations decreases with increasing $P$ : At $B=0.1 \mathrm{~T}$, we find that $88 \%$ of the initial number of $\zeta$ atoms survive for $P=0$, while for $P=1$, this figure is $12 \%$.

The trapped $\zeta$-atom gas will be ultralong lived as, in the zero-temperature limit, two-body collisions can be ruled out because of the Pauli principle. For nonzero temperatures, two-body electronic dipolar relaxation is dominant. Using plane-wave Born expressions ${ }^{12}$ we estimate the corresponding cross section to be $\sigma_{\mathrm{rel}} \simeq(E /$ $\left.E^{\prime}\right)^{1 / 2} \times 10^{-22} \mathrm{~m}^{2}$, with $E\left(E^{\prime}\right)$ the kinetic energy in the initial (final) spin channel. For low collision energies, $E^{\prime}$ tends to a constant yielding the dipolar relaxation rate at low temperatures to be proportional to temperature. For $B=0.1 \mathrm{~T}$, we estimate $G_{\mathrm{dip}} / T=10^{-14} \mathrm{~cm}^{3} \mathrm{~s}^{-1} \mathrm{~K}^{-1}$ $(T \lesssim 0.05 \mathrm{~K})$. Notice that this energy dependence favors relaxation of fast atoms leading to a self-cooling contribution associated with relaxation which is absent in the hydrogen case.

Interestingly enough, though the thermalization rate also vanishes in the low-temperature limit, we found that the system still achieves thermal equilibrium on a time scale substantially smaller than the dipolar lifetime of $\mathrm{D} \uparrow \uparrow$. Thermalization of the trapped $\zeta$ gas may occur through elastic triplet potential scattering or via elastic dipolar collisions. At low temperatures $(T \lesssim 0.03 \mathrm{~K}) \mathrm{di}-$ polar thermalizing collisions dominate because the short-ranged triplet potential becomes ineffective as a result of the Pauli principle. Again, using plane-wave Born expressions, ${ }^{12}$ we estimate the dipolar collision cross section to be $\sigma_{\text {th,dip }}=10^{-22} \mathrm{~m}^{2}$. At higher collision energies, where the Pauli principle becomes less effective, gas-phase thermalization takes place predominantly through elastic scattering via the strong short-ranged triplet potential. A phase-shift analysis yields the corresponding cross section to be proportional to the energy squared: $\sigma_{\text {th,trip }} / E^{2}=10^{-19} \mathrm{~m}^{2} \mathrm{~K}^{2}$. For density $n=10^{14}$ $\mathrm{cm}^{-3}$ and temperature equal to the corresponding Fermi temperature $T_{\mathrm{F}} \simeq 39 \mu \mathrm{K}$, the above expressions yield a lifetime due to dipolar relaxation of several hours and a gas-phase thermalization time of several seconds. Under similar conditions the lifetime of $\mathrm{H} \uparrow \uparrow$ is some seconds. ${ }^{10}$ In contrast to the case of $\mathrm{H} \uparrow \uparrow$ where in the $\operatorname{limit} T \rightarrow 0$ the ratio of the thermalization rate to the relaxation rate vanishes, in the case of $\mathrm{D} \uparrow \uparrow$ this ratio increases as $1 / \sqrt{T}$. This shows the possibility to use evaporative cooling as an efficient means for cooling the trapped gas down to the degeneracy regime.

In the foregoing, degeneracy effects were left out of consideration. An accurate description of such effects depends in a subtle manner on the evaporation scheme and requires a detailed analysis. In a naive picture, the Fermi pressure limits the density for decreasing temperatures, in contrast to the hydrogen case where higher densities are favored, ultimately leading to BEC. In contrast to relaxation, the thermalization rate is affected by blocking effects in the final state. Still, the evaporative cooling scheme may be expected to be very efficient if we take into account that, for low temperatures, the differences in occupation of the single-particle levels, compared with the $T=0$ state, are concentrated at the highest energy levels near the Fermi energy. Cooper pairing in $\mathrm{D} \uparrow \uparrow$ is way out of reach as only $p$-wave pairing is possible, ${ }^{13}$ requiring extremely high densities.

Resonance recombination ${ }^{14}$ and resonance-enhanced relaxation, which are probably the dominant decay mechanisms in magnetically trapped alkalis, are not expected to disturb the above described decay of $\mathrm{D} \uparrow$. The $(v=21, j=0)$ and the $(v=21, j=1)$ molecular levels are just bound, so that resonance recombination can play a role in the decay of $\mathrm{D} \downarrow$. In $\mathrm{D} \uparrow$, however, recombination via these levels is inefficient thanks to the positive sign of the Zeeman energies for the low-field-seeking states. Unbound singlet states also play a negligible role at temperatures of interest as the lowest resonant state $(v=20, j=6)$ is calculated to be $10 \mathrm{~K}$ above threshold. Also the slow decay of $\mathrm{D} \uparrow \uparrow$ is not disturbed by resonance-enhanced processes as collisions proceed via the triplet potential which does not support (almost) bound states.

In the foregoing we discussed the behavior of the trapped gas in some detail, but we did not treat the problem of the loading of the trap and only mentioned some facts relevant to the cooling of the trapped gas. As in the hydrogen case, the development of an efficient filling and cooling scheme is a major project which is left as a challenge to experimentalists.

This work is part of a research program of the Stichting voor Fundamenteel Onderzoek der Materie (FOM), 
which is financially supported by the Nederlandse Organisatie voor Zuiver Wetenschappelijk Onderzoek (ZWO).

${ }^{1}$ T. J. Greytak and D. Kleppner, in New Trends in Atomic Physics, Proceedings of the Les Houches Summer School, 1982, edited by G. Greenberg and R. Stora (North-Holland, Amsterdam, 1984), p. 1125; I. F. Silvera and J. T. M. Walraven, in Progress in Low Temperature Physics, edited by D. Brewer (North-Holland, Amsterdam, 1986), Vol. 10, p. 139; P. J. Nacher, G. Tastevin, M. Leduc, S. B. Crampton, and F. Laloë, J. Phys. Lett. (Paris) 45, L441 (1984), and references therein.

${ }^{2}$ R. Sprik, J. T. M. Walraven, and I. F. Silvera, Phys. Rev. Lett. 51, 479, 942 (1983); H. F. Hess, D. A. Bell, G. P. Kochanski, R. W. Cline, D. Kleppner, and T. J. Greytak, Phys. Rev. Lett. 51, 483 (1983); T. Tommila, S. Jaakkola, M. Krusius, I. Krylov, and E. Tjukanov, Phys. Rev. Lett. 56, 941 (1986).

${ }^{3}$ I. F. Silvera and J. T. M. Walraven, Phys. Rev. Lett. 45, 1268 (1980).

${ }^{4}$ I. Shinkoda, M. W. Reynolds, R. W. Cline, and W. N. Hardy, Phys. Rev. Lett. 57, 1243 (1986).

${ }^{5}$ E. Krotscheck, R. A. Smith, J. W. Clark, and R. M. Panoff,
Phys. Rev. B 24, 6383 (1981); R. M. Panoff, J. W. Clark, M. A. Lee, K. E. Schmidt, M. H. Kalos, and G. V. Chester, Phys. Rev. Lett. 48, 1675 (1982).

${ }^{6} \mathrm{H}$. R. Glyde and S. I. Herandi, in Proceedings of the International Conference on Condensed Matter Theories, San Francisco, 1985, edited by F. B. Malik (Plenum, New York, 1986), Vol. 1, p. 115, and references therein.

${ }^{7}$ C. Lhuillier and F. Laloë, J. Phys. (Paris) 43, 197, 225 (1982), and 44, 1 (1983); E. P. Bashkin and A. E. Meyerovich, Adv. Phys. 30, 1 (1981); W. J. Mullin and K. Miyake, J. Low Temp. Phys. 53, 313 (1983).

${ }^{8}$ D. E. Pritchard, Phys. Rev. Lett. 51, 1336 (1983); H. F. Hess, Phys. Rev. B 34, 3476 (1986); R. V. E. Lovelace, C. Mehanian, T. J. Tommila, and D. M. Lee, Nature (London) 318, 30 (1985); T. J. Tommila, Europhys. Lett. 2, 789 (1986).

${ }^{9}$ A. L. Migdall, J. V. Prodan, W. D. Phillips, T. H. Bergeman, and H. J. Metcalf, Phys. Rev. Lett. 54, 2596 (1985).

${ }^{10}$ A. Lagendijk, I. F. Silvera, and B. J. Verhaar, Phys. Rev. B 33, 626 (1986); H. T. C. Stoof, J. M. V. A. Koelman, and B. J. Verhaar, to be published.

${ }^{11}$ B. J. Verhaar, J. M. V. A. Koelman, H. T. C. Stoof, O. J. Luiten, and S. B. Crampton, Phys. Rev. A 35, 3825 (1987).

${ }^{12}$ J. P. H. W. van den Eijnde, Ph.D. thesis, Eindhoven University of Technology, The Netherlands, 1984 (unpublished).

${ }^{13}$ A. J. Leggett, J. Phys. (Paris), Colloq. 41, C7-19 (1980).

${ }^{14}$ J. Vigué, Phys. Rev. A 34, 4476 (1986). 